\title{
The effect of laser repetition rate on the LASiS synthesis of biocompatible silver nanoparticles in aqueous starch solution
}

This article was published in the following Dove Press journal:

International Journal of Nanomedicine

10 January 2013

Number of times this article has been viewed

\author{
Reza Zamiri' \\ Azmi Zakaria ${ }^{1, *}$ \\ Hossein Abbastabar \\ Ahangar ${ }^{2}$ \\ Majid Darroudi ${ }^{3}$ \\ Golnoosh Zamiri' \\ Zahid Rizwan' \\ Gregor PC Drummen ${ }^{4, *}$ \\ 'Department of Physics, Faculty of \\ Science, Universiti Putra Malaysia, \\ Serdang, Selangor, Malaysia; \\ ${ }^{2}$ Department of Chemistry, Faculty \\ of Science, Universiti Putra \\ Malaysia, Selangor Darul Ehsan, \\ Malaysia; ${ }^{3}$ Advanced Materials and \\ Nanotechnology Laboratory, Institute \\ of Advanced Technology (ITMA), \\ Universiti Putra Malaysia, Serdang, \\ Selangor, Malaysia; ${ }^{4}$ Bionanoscience \\ and Bio-Imaging Program, Cellular \\ Stress and Ageing Program, \\ Bio\&Nano-Solutions, Düsseldorf, \\ Germany \\ *These authors contributed to this \\ work equally
}

Correspondence: Gregor Drummen Helmutstr 3A, D-40472, Düsseldorf, Germany

Tel +492II 22973648

Email gpcdrummen@bionano-solutions.de

\begin{abstract}
Laser ablation-based nanoparticle synthesis in solution is rapidly becoming popular, particularly for potential biomedical and life science applications. This method promises one pot synthesis and concomitant bio-functionalization, is devoid of toxic chemicals, does not require complicated apparatus, can be combined with natural stabilizers, is directly biocompatible, and has high particle size uniformity. Size control and reduction is generally determined by the laser settings; that the size and size distribution scales with laser fluence is well described. Conversely, the effect of the laser repetition rate on the final nanoparticle product in laser ablation is less well-documented, especially in the presence of stabilizers. Here, the influence of the laser repetition rate during laser ablation synthesis of silver nanoparticles in the presence of starch as a stabilizer was investigated. The increment of the repetition rate does not negatively influence the ablation efficiency, but rather shows increased productivity, causes a red-shift in the plasmon resonance peak of the silver-starch nanoparticles, an increase in mean particle size and size distribution, and a distinct lack of agglomerate formation. Optimal results were achieved at $10 \mathrm{~Hz}$ repetition rate, with a mean particle size of $\sim 10 \mathrm{~nm}$ and a bandwidth of $\sim 6 \mathrm{~nm}$ 'full width at half maximum' (FWHM). Stability measurements showed no significant changes in mean particle size or agglomeration or even flocculation. However, zeta potential measurements showed that optimal double layer charge is achieved at $30 \mathrm{~Hz}$. Consequently, Ag-NP synthesis via the laser ablation synthesis in solution (LASiS) method in starch solution seems to be a trade-off between small size and narrow size distributions and inherent and long-term stability.
\end{abstract}

Keywords: laser ablation, repetition rate, silver nanoparticle, starch, amylose, amylopectin

\section{Introduction}

Over the past 20 years, engineered nanoparticles have increasingly been the focus of attention and intense research in various fields of science and engineering. These particles with nanometer-scale dimensions, typically smaller than $100 \mathrm{~nm}$, have become important materials in a myriad of applications, eg, as imaging agents in biological and (bio)medical research; as drug delivery vehicles in (bio)medicine; as constituents of paints, coatings, and pastes; in electronics and photovoltaic applications; and in LEDs, lasers and many other advanced technologies.

At the mesoscopic size range, nanoparticles display unique and divergent optical, electronic, thermal, magnetic, and structural properties compared with their bulk material counterparts. ${ }^{1-5}$ These properties are largely size and shape dependent and are the result of strict confinement of electrons and holes so that their energy levels are no longer continuous, but discrete and quantized (quantum confinement). Collectively, the aforementioned properties and their large surface-to-volume ratios open up new 
areas and opportunities for their utilization, in particular in biomedical and electronic applications. Recently, Roduner intelligibly and comprehensively described why size and shape matter and why nanoparticles are so distinctly different. $^{4}$

Metal nanoparticles (MNPs) represent an important class of materials with unprecedented and specific properties, and are particularly interesting because they display various plasmon phenomena - a quantum of plasma oscillations, which occurs when light radiation interacts with free electrons in a plasmonic nanoparticle $\mathrm{e}^{1,2}$ - which can be exploited particularly for (bio)imaging and other optical applications. In silver nanoparticles (Ag-NPs), the surface plasmon resonance (SPR) is size and shape dependent, and its energy is located far from the interband transition energy, which is a major advantage compared with other MNPs. ${ }^{6}$ Furthermore, noble MNPs display SPR in the visible region of the electromagnetic spectrum and red-shift their wavelengths as the particle size increases. $^{7,8}$

Metal nanoparticles can be synthesized by a variety of physical and chemical methods, which can be broadly categorized into wet chemical methods, ion implantation, and chemical or physical vapor deposition, each of which has its own distinct advantages and disadvantages. Silver nanoparticles specifically can be synthesized chemically by reduction of $\mathrm{AgNO}_{3}$ with sodium citrate, ${ }^{9}$ but this method is disadvantageous because of surface fouling by residual citrate ions, or by alternative means such as the recently proposed chemical reduction-protection method, involving a mixture of silver-ammonia complex, sodium borohydride, and lauric acid. ${ }^{10}$ The tendency of MNPs to agglomerate is an additional major drawback for a variety of applications and the (semi) product's shelf-life.

Analogous to laser-induced preparation of gas-phase clusters, ${ }^{11}$ a laser ablation method in liquid was developed for the synthesis of MNPs in the groups of Henglein and Cotton. ${ }^{12-14}$ Laser ablation synthesis in solution (LASiS) is an attractive alternative to the abovementioned methods and is compelling because of its uncomplicated and straightforward experimental setup and synthesis procedure. ${ }^{12-15}$ Furthermore, the preparation of MNPs by LASiS is devoid of surface contamination with residual reactants and concomitant potential cytotoxic effects. ${ }^{12}$ Nonetheless, nanoparticles synthesized via LASiS are still subject to the same propensity to agglomeration as their chemically produced counterparts, albeit to a lesser extent. Therefore, the major challenge in MNP synthesis is to devise methods that allow efficient synthesis without the need for further purification, produce nanoparticles that can directly be utilized or easily functionalized for biomedical applications, and that have significantly reduced tendencies to precipitate or flocculate. Recently, natural materials such as long-chain fatty acids (stearic, palmitic, and lauric acid), lauryl amine, ${ }^{16}$ and chitosan ${ }^{17}$ have been used as stabilizers in the synthesis of MNPs. These stabilizing agents prevent particle agglomeration by physically surrounding the MNPs (capping) resulting in charge and/ or steric stabilization.

Comparable to the aforementioned stabilizing methods, we previously described the successful preparation of silver nanoparticles in an aqueous solution of starch by laser ablation of a silver plate. ${ }^{18}$ Starch is a natural, renewable, and biodegradable polymer that can be used as a suitable dispersant or stabilizer for the preparation of nanoscale metal particles ${ }^{18-20}$ and would not endanger LASiS' reputation as a 'green' synthesis technique. In addition, several enzymes, such as $\alpha$ - and $\beta$-amylase and amyloglucosidase, exist that are capable of digesting the starch polymer and releasing the nanoparticle, ${ }^{21}$ which is useful in targeted and controlled delivery applications in (bio)medicine.

Our previous results showed that starch plays a dual role in this synthesis process, ie, as a reducing and capping agent, prevents agglomeration, stabilizes the nanoparticle for prolonged periods of time, and allows some control over the final mean particle size. However, it is increasingly clear that the ablation conditions markedly influence the ablation efficiency and the mean size and size distribution of the final product. Understanding the conditions and basic colloid formation mechanism involved in LASiS may possibly result in an uncomplicated, green, nontoxic method with acceptable size control and easy biofunctionalization. This paper expands our previous research on starch-stabilized Ag-NPs by closely investigating the effect of laser repetition rate on the produced nanoparticles to determine whether better control over size and shape is possible.

\section{Experimental procedures}

Laser ablation procedures were essentially performed as described previously, ${ }^{18}$ with minor adaptations. Briefly, a 99.99\% pure silver plate (Sigma-Aldrich, Singapore) was first cleaned in an ultrasonic bath for 30 minutes to remove surface impurities. Subsequently, the plate was suspended in a cubic glass cell, close to the cell wall (Figure 1) that contained $20 \mathrm{~mL}$ of freshly prepared aqueous starch solution (1\% wt). The solution was continuously stirred with a magnetic stirring 

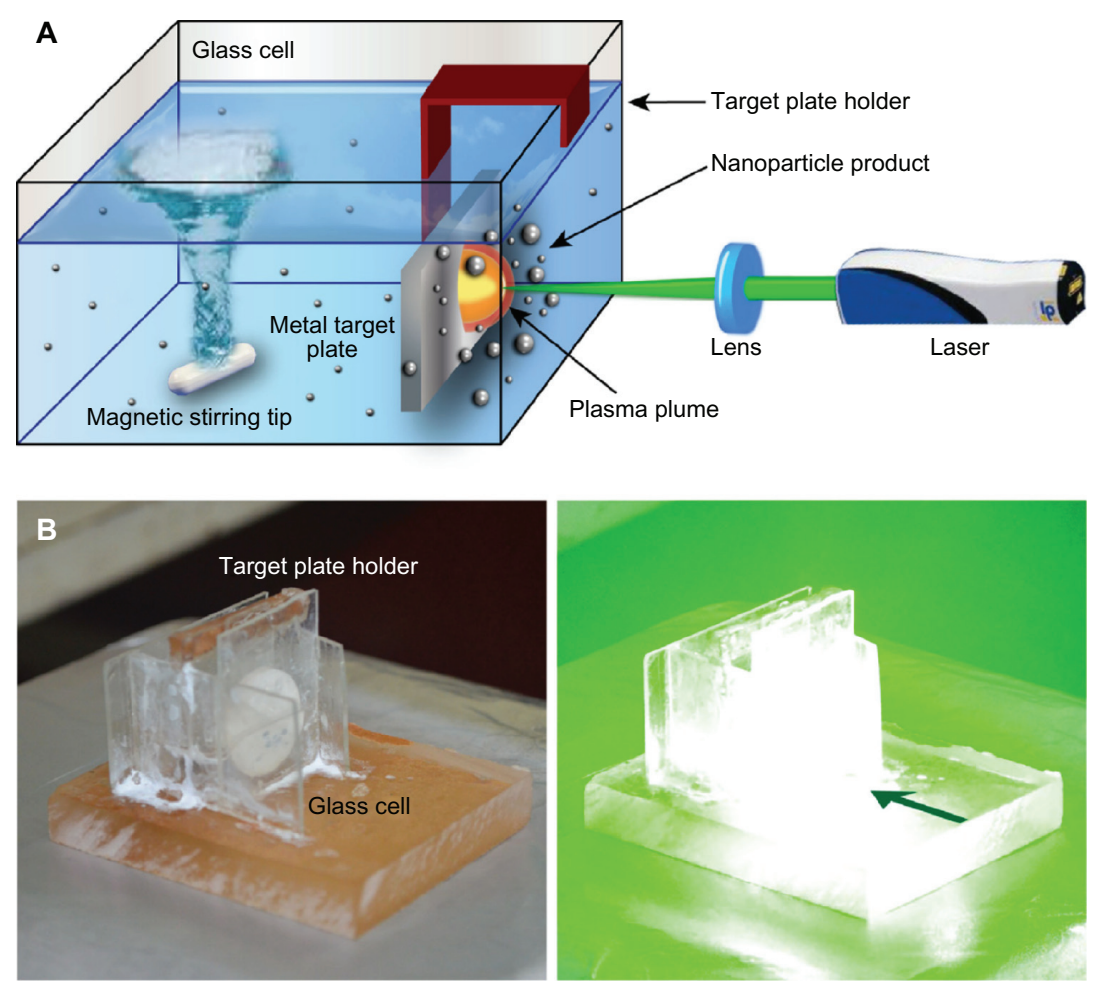

Figure I Laser ablation synthesis in solution (LASiS). (A) Schematic representation of the experimental setup for laser ablation; (B) experimental LASiS setup, with a target plate placed close to the cell wall.

Note: The green arrow indicates the direction of the laser beam.

device at ambient temperature to disperse the produced AgNPs. A pulsed Q-Switched Nd:YAG laser (Spectron SL400/ SL800 system; GSI Lasers, Rugby, UK) with variable repetition rate and $10 \mathrm{~ns}$ pulse duration at its second harmonic wavelength (532 nm) was used to prepare the Ag-NPs. The laser output power of $60 \mathrm{~mJ} /$ pulse was monitored with an optical power detector. The Nd:YAG laser beam was focused at the silver target using a $25-\mathrm{cm}$ focal length lens. The ablation was performed for 15 minutes at different laser repetition rates, ie, 10, 20, 30, and $40 \mathrm{~Hz}$, respectively.

The prepared Ag-NPs were characterized with an UV-vis double beam spectrophotometer (Shimadzu, Columbia, MD, USA) and a Hitachi H-7100 transmission electron microscope (TEM; Hitachi, Chula Vista, CA, USA) operating at an accelerating voltage of $120 \mathrm{kV}$. The samples were prepared for TEM experiments by depositing a drop of solution containing Ag-NPs onto carbon-coated copper grids and left to dry for 1 day at ambient temperature. An atomic absorption spectrometer (AAS-S Series; Thermo Scientific, San Jose, CA, USA) was used to measure total silver in the starch solution and the calibration of the AAS was performed with a standard certified AAS silver solution (Inorganic Ventures, Christiansburg, VA, USA). Zeta potentials of the prepared samples were measured with a Zetasizer 3000HS photon correlation spectrometer (Malvern Instruments Ltd, Malvern, UK) at an applied voltage of $100 \mathrm{~V}$.

The nanoparticle size distribution was analyzed with UTHSCSA ImageTool (v 3). Standard procedures were used to calculate means and standard deviations using SPSS statistics software (v 17; SPSS, Inc, Chicago, IL, USA). Curve-fitting was performed with IgorPro 5 (Wavemetrics, Lake Oswego, OR, USA). Experiments were repeated with distilled water as a solvent under similar conditions for reference purposes.

\section{Results and discussion}

In this series of experiments, we aimed to control the nanoparticle size and size distribution by using both starch as a stabilizer and varying the repetition rate. In order to obtain fast particle growth from the initial nucleation, the experimental setup was chosen accordingly, ie, the plate was placed close to the container's wall as depicted in Figure 1. Furthermore, the distance from the cell wall was large enough to avoid any deposition of Ag-NPs on the cell wall (Figure 1B), as previously utilized by Yang et al to form surface-enhanced Raman scattering glass substrates. ${ }^{22}$ In this configuration, the stirring device dispersed formed nanoparticles, but direct turbulence near the plate and plasma 
plume was avoided. We hypothesized that in this way, a more continuous particle growth might be achieved rather than a reduction of the cluster size through fragmentation.

At the beginning of the laser ablation process, the starch solution appeared fully transparent and turned to a clear light yellow at low repetition rates and an increasingly intense dark yellow for higher repetition rates. The darker yellow color is indicative of high Ag-NP concentrations, which was verified by the increase of the intensity of the plasmon absorption band in the recorded UV-vis absorption spectra (Figure 2). Furthermore, the change in color of the solution was faster for higher repetition rates compared to lower repetition rates at the same laser intensity of $60 \mathrm{~mJ} /$ pulse.

The absorption maxima in the spectra of Ag-NP colloids in starch solution ( $1 \% \mathrm{wt}$ ) at laser repetition rates incrementally increasing from 10 to $40 \mathrm{~Hz}$ are shown in Figure 2.
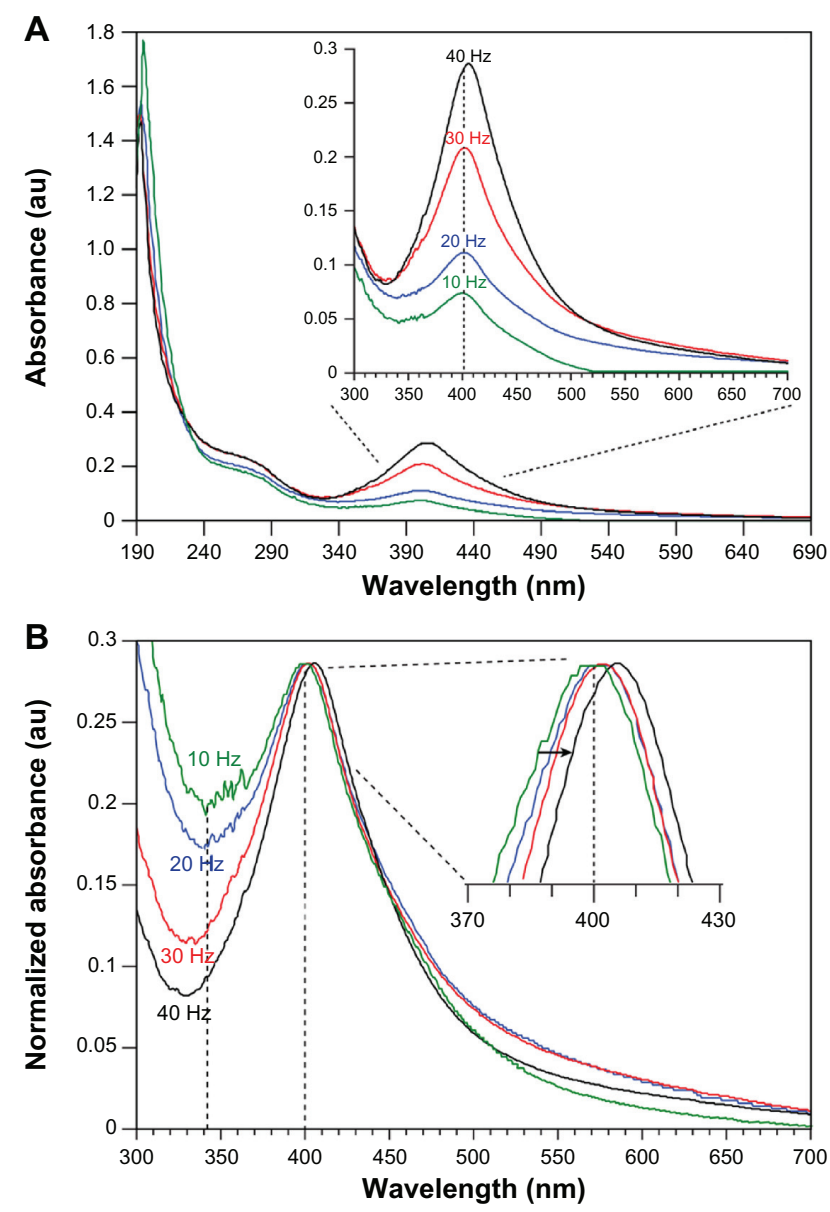

Figure 2 UV-vis absorption spectra (A) and normalized spectra (B) of Ag-NP samples prepared at different laser repetition rates in aqueous starch solution (ablation time $=15$ minutes; $\lambda_{\text {max }}=402 \mathrm{~nm}$ ). With increasing repetition rate, a clear increase is seen in the maximal absorbance $(\mathbf{A})$, which indicates an increase in $\mathrm{Ag}-\mathrm{NP}$ content. The insert in (B) shows a red-shift with increasing repetition rate in conformity with an increase in mean particle size (normalized to $\lambda_{\text {max }-40 \mathrm{~Hz}}$ for comparison reasons).

Abbreviation: Ag-NP, silver nanoparticle.
Generally, Ag-NP spectra are characterized by their size and shape $^{7,8}$ and the interparticle distance within agglomerates. ${ }^{23}$ The spectra in Figure 2A show a symmetrical and narrow peak at about $400 \mathrm{~nm}$ and a broad tail in the UV part, which are both characteristic of $\mathrm{Ag}(0)$ particles, as extensively described by Kerker $^{24}$ and more recently by Kleinermanns et al, ${ }^{25}$ and originate from particle plasmon excitation and interband transitions. Since the particle size determines the absorption wavelength, ${ }^{7}$ the blue shift of the absorption maximum in the order of 20-25 nm compared with absorption maxima reported for similar colloids produced via chemical reduction indicates a considerably smaller average particle size. Furthermore, the bandwidth of the electronic absorption at the highest repetition rate $(40 \mathrm{~Hz})$ was significantly smaller than chemically produced colloids, ie, $87 \mathrm{~nm}$ compared to a bandwidth of $\sim 162 \mathrm{~nm},{ }^{25}$ which altogether indicates that LASiS produces a narrower particle size distribution per se. The prominent single peak at about $400 \mathrm{~nm}$ suggests that the Ag-NPs present in the starch solution were predominantly spherical in shape, as described in various reports. ${ }^{23-25}$ The spherical shape was further corroborated visually by TEM evaluation as shown in Figure 3. The spectra in Figure 2A clearly show that the absorption peak intensity increases with the increment of the laser repetition rate, which suggests that the particle density increased in the solution. Logically, with increasing repetition rate, the number of ablation events increases and thus the number of particles generated. Regression analysis of the repetition rate versus maximal peak absorption showed a near linear increase $\left(R^{2}=0.987\right)$. This might be expected since at constant pulse energy, the laser power scales with the repetition rate.

To verify these results, atomic absorption spectroscopy (AAS) was performed and showed that with increasing repetition rate, the density increased from $0.37 \pm 0.02,0.56 \pm$ 0.05 , and $0.84 \pm 0.09$ to $1.06 \pm 0.04 \mathrm{mg} / \mathrm{L}$. Linear regression analysis indicated that the nanoparticle content increased near linearly $\left(\mathrm{R}^{2}=0.9951\right)$ with the repetition rate, which is in good agreement with the UV-vis results and the visual observation of rapid color change (vide supra).

From the insert in Figure 2B it becomes clear that a shift in the spectra towards longer wavelengths with increasing repetition rate occurred, which may be attributed to an increase in mean particle size. Furthermore, the long wavelength tail $(>450 \mathrm{~nm})$ also increases with increasing repetition rate (insert Figure 2A). These observations are in contrast to results reported by Mafuné et $\mathrm{al}^{26}$ and by Menéndez-Manjón and Barcikowski ${ }^{27}$ for LASiS of gold nanoparticles in aqueous surfactant solution or de-ionized water. In particular, Menéndez- 

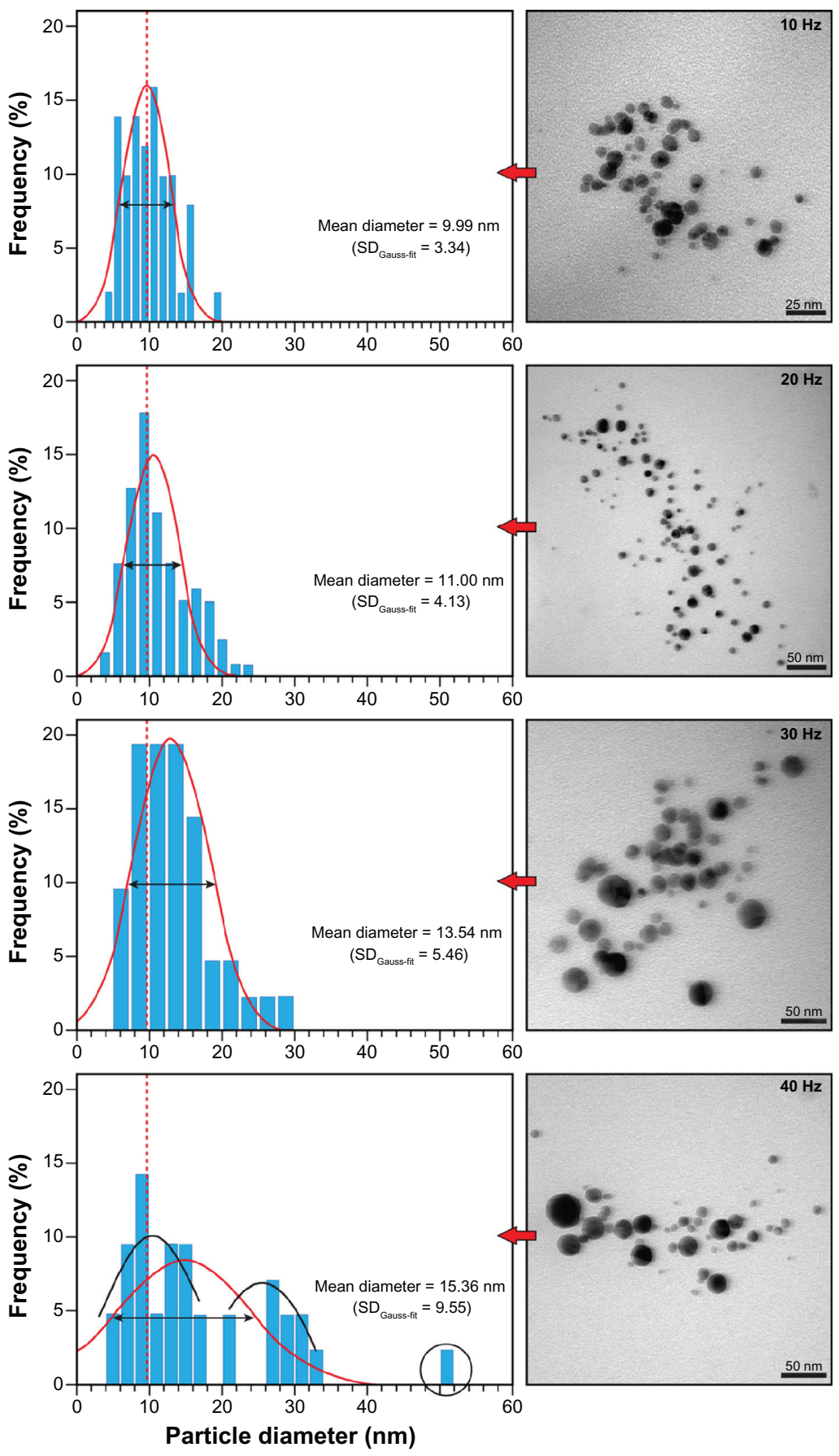

Figure 3 Representative TEM images and size distributions of silver nanoparticles prepared by $532 \mathrm{~nm}$ LASiS (60 mJ/pulse; $10 \mathrm{~ns}$ ) in an aqueous starch solution (I\% wt) with the indicated repetition rates (standard deviations in the normal distribution in parenthesis).

Notes: At $40 \mathrm{~Hz}$, essentially two populations exist (black Gaussian curves) and occasionally ( $<3 \%$ ) larger particles of $\sim 50 \mathrm{~nm}$ (circle).

Abbreviations: LASiS, laser ablation synthesis in solution; TEM, transmission electron microscope.

Manjón and Barcikowski reported that higher laser repetition rates resulted in colloid fragmentation and a decreasing mean particle size and distribution. However, they use significantly higher repetition rates over a larger range $(100-5000 \mathrm{~Hz})$ and an experimental setup that promotes fragmentation. Intuitively, in a setup in which the plate is attached to the wall and the laser beam passes through the bulk of the solution, a higher repetition rate means that colloidal clusters are 'hit' more frequently by the laser pulse per unit time and thus the clusters absorb more photon energy and therefore must be fragmented more compared with the effect at lower repetition rates. Stirring such a colloidal solution during the ablation procedure, to introduce turbulence, might more efficiently exchange particles from fragmented clusters with fresh intact 
clusters in the irradiated zone. In fact, Menéndez-Manjón and Barcikowski recently proposed that even in a more or less stagnant liquid zone, the particle population in the irradiated zone is efficiently replenished between consecutive pulses, due to convective-diffusion. ${ }^{27}$ Our results show a restricted fragmentation of the colloidal particles, which we attribute to the current experimental setup in which the metal plate was placed close to the cell wall and the laser was focused onto the plate via the shortest path through the solution (Figure 1). This setup in combination with starch and its inherent properties might allow more controlled crystal growth and abrogate the formation of the clusters to begin with.

To further evaluate these assumptions, TEM imaging was used to determine the mean particle size and size distribution and to evaluate the morphology of the individual particles and possible aggregates. As stated previously, predominantly spherical particles were observed (see inserts Figure 3). However, with increasing repetition rate, visual evaluation on TEM appeared to indicate that larger spherical particles dominated the number of clusters formed. Generally, fewer clusters were observed compared with other LASiS methods ${ }^{28,29}$ or LASiS in distilled water (vide infra). At the lowest repetition rate of $10 \mathrm{~Hz}$, the smallest average size of $\sim 10 \mathrm{~nm}$ was recorded with a narrow size distribution. No particles below $\sim 5 \mathrm{~nm}$ were observed, but at the $40 \mathrm{~Hz}$ repetition rate, larger globular particles of more than $50 \mathrm{~nm}$ were frequently detected.

Gaussian fitting of the size distribution, as shown in Figure 3, revealed that the mean particle size increased nearly linearly with the repetition rate (see also Figure 4), whilst the number of Ag-NPs with sizes smaller than $10 \mathrm{~nm}$ dropped from $45.5 \% \pm 3.6 \%$ at $10 \mathrm{~Hz}$ to $15.1 \% \pm 3.9 \%$ at $40 \mathrm{~Hz}$. Furthermore, the broadening in 'full width at half maximum' (FWHM) and the increasing standard deviation in the Gaussian distribution ( $\mathrm{SD}_{\text {Gauss-fit }}$ ) in Figure 3 overall substantiate the spectroscopic results, implying both the formation of larger particles and a higher size variation within the nanoparticle population with increasing repetition rate. The results of multiple measurements are depicted in Figure 4.

Control experiments in double distilled water were performed under the same experimental conditions for comparison. The dispersion of Ag-NPs prepared in starch solution was clearer compared to the dispersion in distilled water, which presented itself as a somewhat opaque solution. In conformity to LASiS in starch solution, the absorption increased with increasing repetition rate, as shown in Figure 5. The absorption maximum for Ag-NPs formed at the lowest repetition rate of $10 \mathrm{~Hz}$ was located at $393 \mathrm{~nm}$, which is a difference of $9 \mathrm{~nm}$ to the same LASiS in starch solution (403 nm; Figure 2) and shows

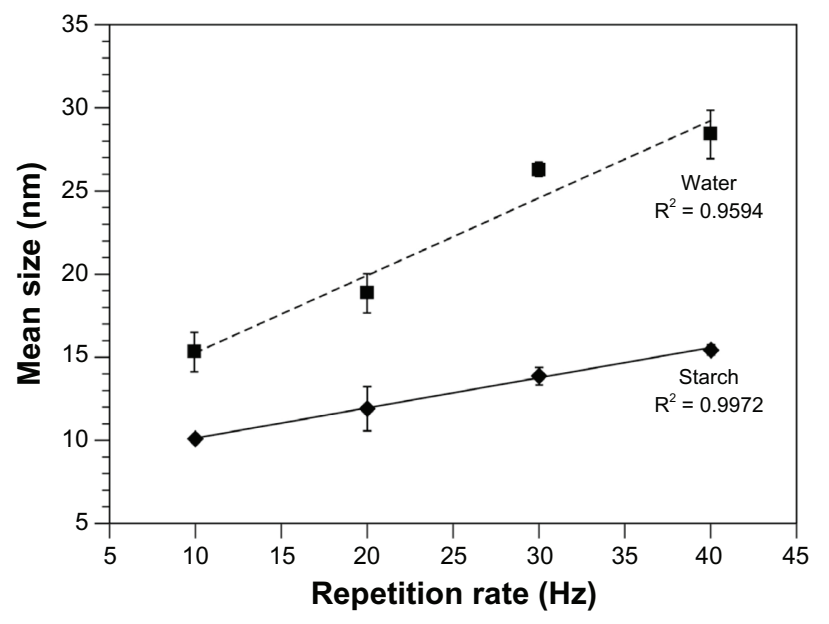

Figure 4 Mean $\mathrm{Ag}-\mathrm{NP}$ size as a function of the repetition rate. Results were calculated from TEM images and size distributions of silver nanoparticles prepared by $532 \mathrm{~nm}$ LASiS (60 mJ/pulse; $10 \mathrm{~ns})$ in an aqueous starch solution (I\% wt) or distilled water.

Note: Error bars reflect the SD of multiple measurements; $n=3$.

Abbreviations: Ag-NP, silver nanoparticles; LASiS, laser ablation synthesis in solution; SD, standard deviation; TEM, transmission electron microscope.

that initially smaller nanoparticles were formed. However, the total shift to $403 \mathrm{~nm}$ at $40 \mathrm{~Hz}$ repetition rate is twice that of a comparable LASiS in starch solution (400 to $405 \mathrm{~nm}$ ). In addition, the long wavelength tail shows a significantly higher absorption than starch LASiS (compare Figures 2A and 5), which indicates the presence of agglomerates that absorb at higher wavelengths. The peaks themselves appear broader compared with those in starch LASiS, indicating a broader Ag-NP size distribution, which was subsequently confirmed by TEM evaluation (data not shown).

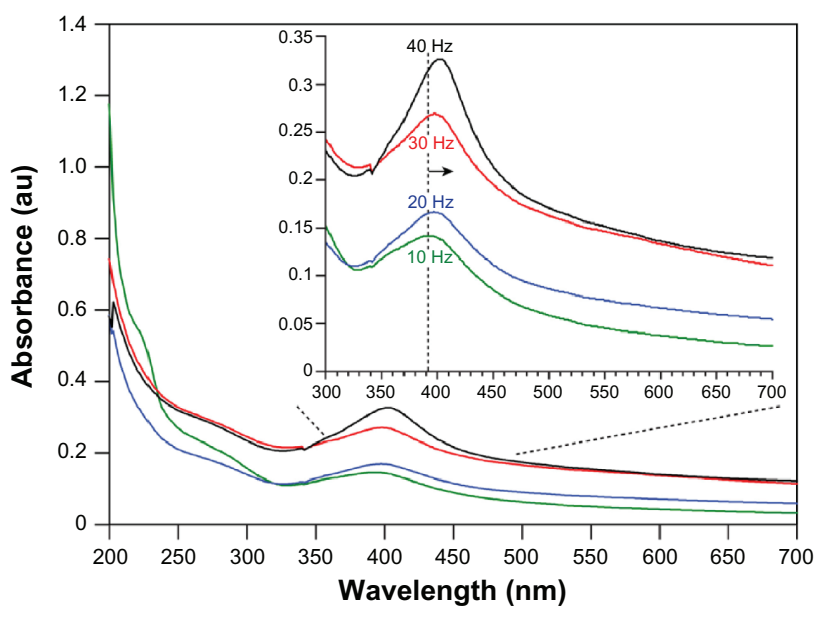

Figure 5 UV-vis absorption spectra of Ag-NP samples prepared by $532 \mathrm{~nm}$ LASiS $(60 \mathrm{~mJ} /$ pulse; $10 \mathrm{~ns})$ in distilled water at different laser repetition rates (ablation time $=15$ minutes; $\lambda_{\max }=393 \mathrm{~nm}$ ). With increasing repetition rate, a clear increase in maximal absorbance is noticeable, which indicates an increase in Ag-NP content. Furthermore, a clear red-shift of $\lambda_{\text {max }}$ is noticeable in the insert, which is in conformity with an increase in mean particle size.

Abbreviations: $\mathrm{Ag}-\mathrm{NP}$, silver nanoparticles; LASiS, laser ablation synthesis in solution. 
TEM evaluation revealed that starch LASiS produced spherical particles, whereas LASiS in water resulted in semispherical and more erratic particles (Figure 6A). Furthermore, with increasing repetition rate, increased cluster formation and deviation from sphericity was observed, which is in good agreement with the spectroscopic results. The mean particle size at $40 \mathrm{~Hz}$ repetition rate was $30.8 \pm 3.9 \mathrm{~nm}$ (in the single measurement in Figure 6B: $27.91 \mathrm{~nm}$ ) and contrary to the observations in starch solution, several large clusters of at least $230 \mathrm{~nm}$ in diameter were observed (Figure 6B). Moreover, visual comparison of Figures 3 and 6 shows that the particle size was considerably smaller in the starch solution compared to that in distilled water. Indeed, the mean particle size for LASiS in water was significantly larger than for starch LASiS and the increase in mean particle size scaled at least seven times faster with the repetition rate, as deduced from the slopes in Figure 4. These results are generally in good agreement with our previous observations ${ }^{18,30}$ and the results reported by others. ${ }^{26,31}$

$\mathrm{Ag}-\mathrm{NPs}$ are generally formed through phase transition, followed by nucleation during plasma plume cooling for which the driving force is supersaturation, and further crystal growth. ${ }^{31,32}$ The embryonic particle grows by attracting emitted material, such as silver atoms, clusters, and droplets released from the silver plate during the laser ablation process, which is a diffusion-limited process. Furthermore, clusters of various sizes can be formed by coalescence of individual growing embryonic particles. In the presence of a stabilizing molecule, the expansion rate of the particle is limited and finally halted as the stabilizing molecules increasingly cover the particle surface (Figure 7) thereby preventing processes such as nuclei coalescence or adsorption of free ions and atoms from the solvent vicinity. As a result, the mean size must be much smaller compared with

\section{LASiS in water.}

The two major macromolecular components of starch $\left[\left(\mathrm{C}_{6} \mathrm{H}_{10} \mathrm{O}_{5}\right)_{\mathrm{n}}\right]$ are the linear $\alpha$-1,4-D-glucopyranose residues linked amylose, which adopts a left-handed helical conformation in watery solutions (Figure 7A), and the branched amylopectin, which consists of short branches of $\alpha-1,4-\mathrm{D}-$ glucopyranose that are interlinked by $\alpha-1,6-\mathrm{D}$-glycosidic linkages. Most common types of starch contain $70 \%-80 \%$

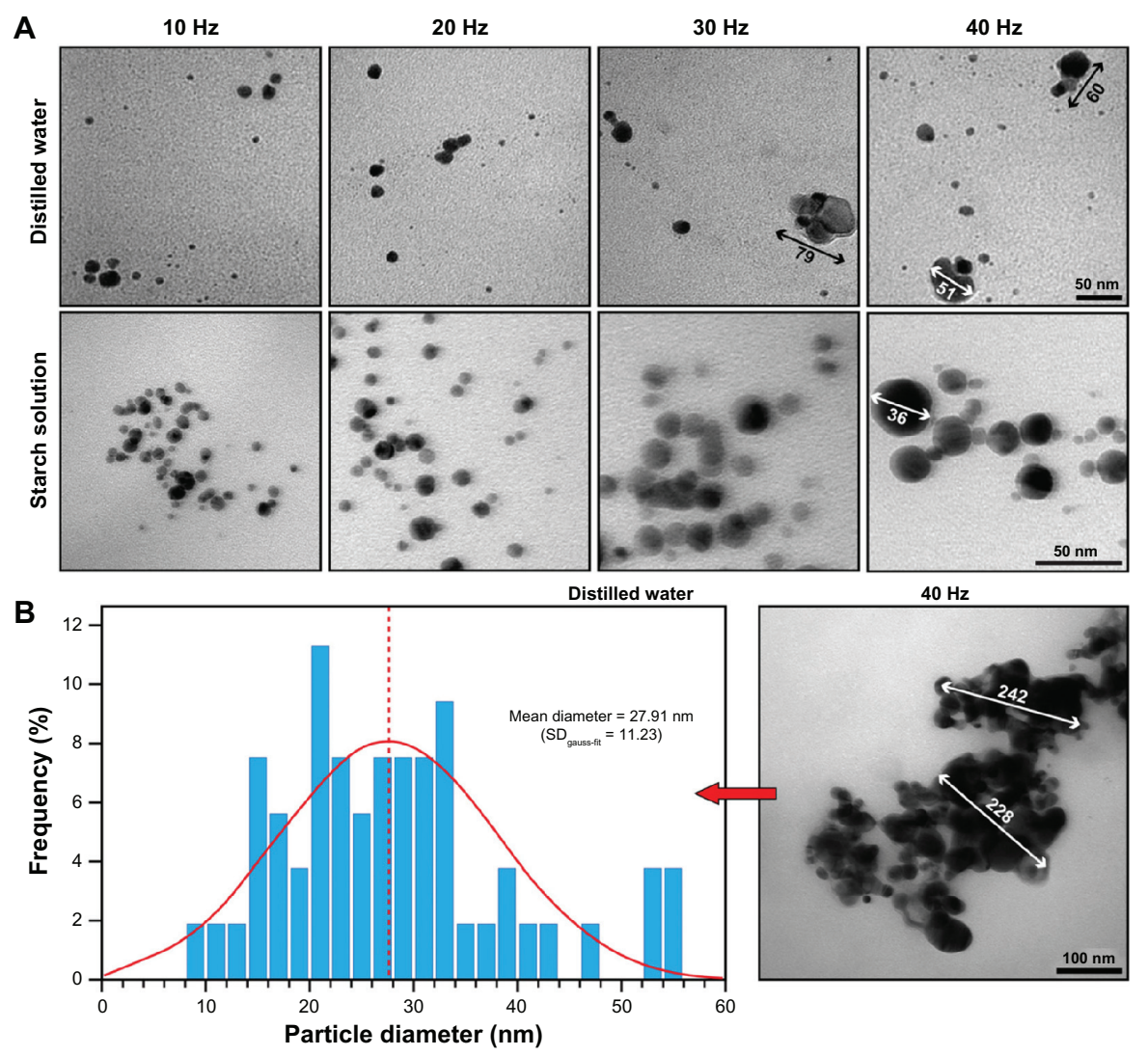

Figure 6 (A) Representative TEM images of silver nanoparticles prepared by $532 \mathrm{~nm}$ LASiS (ablation time $15 \mathrm{minutes} ; 60 \mathrm{~mJ} / \mathrm{pulse}$; $10 \mathrm{~ns}$ ) in an aqueous starch solution $(1 \% \mathrm{wt})$ or distilled water with the indicated repetition rates. Notice the significant deviation from sphericity and agglomeration of particles in LASiS in water; (B) mean size and size distribution in water at $40 \mathrm{~Hz}$ repetition rate. Here, several large clusters of agglomerated particles were observed.

Abbreviations: LASiS, laser ablation synthesis in solution; TEM, transmission electron microscope. 
amylopectin and 20\%-30\% amylose, ${ }^{33}$ depending on their botanical origin. Because of their different structures and branching, amylose and amylopectin behave differently in aqueous solution. It is well established that amylose forms single helical inclusion complexes with suitable molecules. ${ }^{34,35}$

As early as 2002, Stoddart's group showed that singlewalled carbon nanotubes (SWCNTs), which are normally insoluble in water, can be solubilized in aqueous starch solution because amylose wraps itself helically around SWCNTs. ${ }^{36}$ This was achieved by displacing iodine ions (commonly used to spectroscopically determine the amylose content of starch by forming polyiodide chains inside the helix composed of $\mathrm{I}_{3}^{-}$and $\mathrm{I}_{5}^{-}$subunits) during sonication from the helix. Chattopadhyay et al recently showed that treatment of a gold $\mathrm{Au}-\mathrm{NP}-$ starch composite $(10-30 \mathrm{~nm})$ with $\alpha$-amylase led to the degradation of starch into its lower analogs, which resulted in the release of the encapsulated $\mathrm{Au}-\mathrm{NPs} .{ }^{21}$ The cavity dimension of the amylose helix is believed to be variable and adapts itself to accommodate differently proportioned particles. Thus, Ag-NPs might be present in a helical starch complex as depicted in Figure 7A, but this hypothesis requires further experimental research. Alternatively, since the amylopectin content is much higher, the extensive network of hydrogen bond-forming groups may interact with the surface of the Ag-NPs as shown in Figure 7B. The result is a dendrimer-like corona, which also leads to surface passivation or protection against nanoparticle aggregation by reduction of the surface energy, chemical reactivity, and steric hindrance. Interestingly, Stoddart and co-workers noticed that in the absence of amylopectin, the

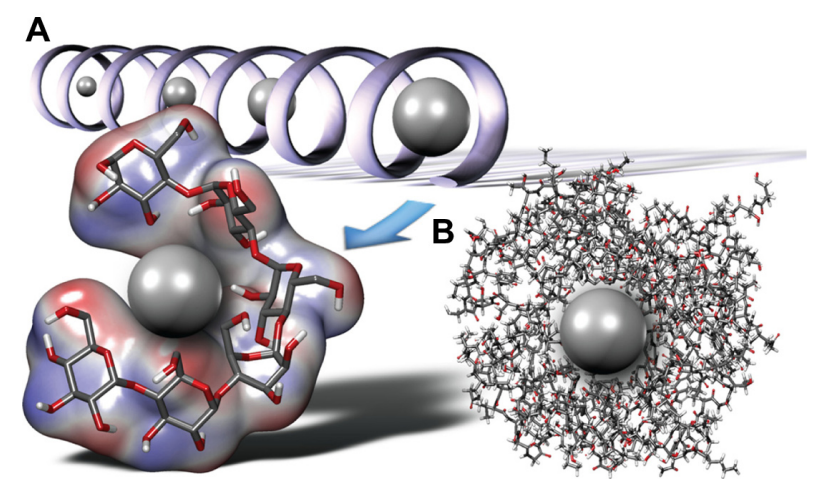

Figure 7 Schematic and hypothetical representation of individual starch components interacting with the LASiS-produced Ag-NPs. (A) Particle growth in the left-hand helical amylose, with the helical structure adjusting itself to the growing particle. Extensive interaction via hydrogen bonds with the Ag-NP surface may occur, thereby passivating and shielding the $\mathrm{Ag}-\mathrm{NP}$; (B) extensive network of highly branched amylopectin surrounds and shields the Ag-NP. In natural starch in which both amylopectin and amylose are present, combinations of these models may be expected.

Abbreviations: $\mathrm{Ag}-\mathrm{NP}$, silver nanoparticles; LASiS, laser ablation synthesis in solution.
SWCNT-amylose composite became insoluble in water, ${ }^{36}$ which suggests that a hybrid of both forms is present, ie, a NP-loaded amylose helix, coated with amylopectin.

To determine the stability of the Ag-NP-starch composite in solution, samples previously prepared and stored for 1 month in the dark at ambient temperature were reevaluated by recording UV-vis spectra (Figure 8). By comparing the spectra of the freshly prepared Ag-NPs (dotted lines) with those recorded after 1 month in Figure 8A, it becomes clear that the long wavelength tail (400 to $800 \mathrm{~nm}$ ) increases upon prolonged storage and particle aging. Such increases are normally attributed to the formation of larger clusters. Analysis of the enclosed area-under-the-curve (AUC) according to: $\triangle \mathrm{AUC}=\mathrm{AUC}_{0 \text {-days }}-\mathrm{AUC}_{30 \text {-days }}$ shows that with increas-
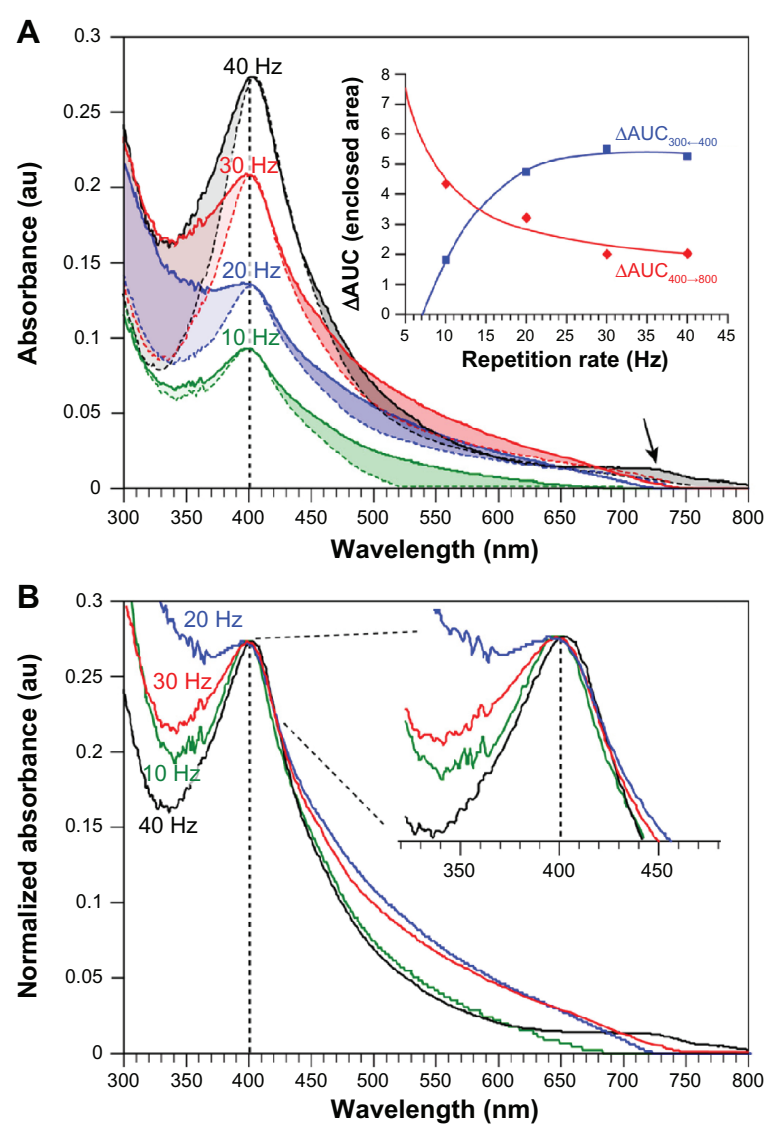

Figure 8 UV-vis absorption spectra (A) and normalized spectra (B) of freshly prepared Ag-NP samples (dotted lines) and samples stored for I month in the dark at ambient temperature (solid lines).

Notes: Samples were prepared by $532 \mathrm{~nm}$ LASiS $(60 \mathrm{~mJ} / \mathrm{pulse} ; 10 \mathrm{~ns})$ in an aqueous starch solution ( $1 \% \mathrm{wt}$ ) at different laser repetition rates (ablation time $=15$ minutes; $\lambda_{\max }=403 \mathrm{~nm}$ ). (A) An increase in absorption of the long wavelength tail can be clearly noticed for all repetition rates, as well as a clear pool of particles with a $\lambda_{\max -40 \mathrm{~Hz}}=723 \mathrm{~nm}$ at $40 \mathrm{~Hz}$ repetition rate (black arrow). Furthermore, a characteristic change below $400 \mathrm{~nm}$ occurs. The changes in the enclosed areas were calculated via integration and subtracting spectra from 30-day-old samples from newly prepared solutions (AUC). The insert in (B) shows no significant red-shift with increasing storage (spectra normalized to $\lambda_{\max -40 \mathrm{~Hz}}$ for comparison reasons).

Abbreviations: AUC, area under the curve; LASiS, laser ablation synthesis in solution. 
ing repetition rate, the increase in the long wavelength tail becomes smaller (Figure 8A insert). For $40 \mathrm{~Hz}$ repetition rate, a shoulder at $730 \mathrm{~nm}$ may be noticed, which might indicate the formation of a particular pool of clusters or elongated particles, since these normally show a second plasmon peak at $>700 \mathrm{~nm}$. Figure $8 \mathrm{~A}$ also reveals that the UV part of the spectrum $(<400 \mathrm{~nm})$ changes markedly and shows a reciprocal behavior compared with the long wavelength tail in that it increases noticeably with repetition rate (see insert). Even though various changes in the spectra may be observed, Figure 8B shows that the absorption maximum at $400 \mathrm{~nm}$ does not shift significantly (see inserted enlargement), which indicates that the mean particle size does not change. Evaluation using TEM of the stored solutions indeed confirmed this. For instance, the mean particle size of a 1-month-old Ag-NP solution prepared at $10 \mathrm{~Hz}$ was
$12.0 \pm 1.2 \mathrm{~nm}$ compared with $10.1 \pm 0.2 \mathrm{~nm}$ for a freshly prepared solution, which is an insignificant increase. Overall, these results suggest that after 30 days of storage, some minor deterioration of the $\mathrm{Ag}-\mathrm{NP}$ solution might occur, but not necessarily major agglomeration effects.

Finally, zeta potential measurements were performed to further assess the stability of the Ag-NP-starch composite in solution or changes thereof over time. The results presented in Figure 9 show some interesting phenomena. First, from Figure 9A it may be deduced that both in pure water and in aqueous starch solution, the zeta potential varies with the repetition rate and reaches an apex of 20 and $30 \mathrm{~Hz}$ for water and aqueous starch, respectively. Second, the Ag-NPs are more stabile in the presence of starch compared with pure water. Third, with the exception of $10 \mathrm{~Hz}$, a good-to-excellent stability of the Ag-NP solution is reached
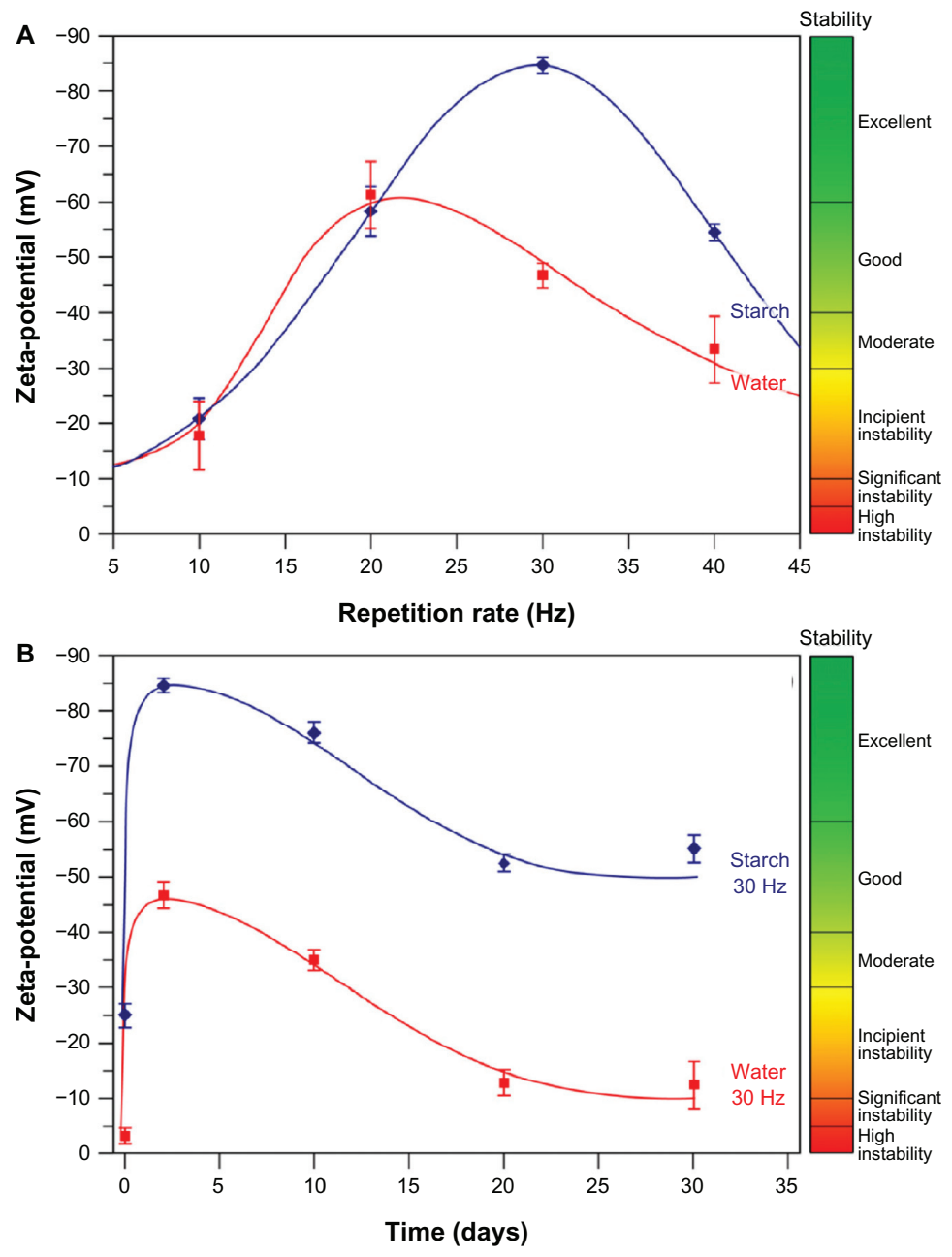

Figure 9 Zeta potential determination ${ }^{38,39}$ to evaluate $\mathrm{Ag}-\mathrm{NP}$ stability over time. (A) Zeta potential varies with the repetition rate as measured after 24 hours. Maximal stability was achieved with the $30 \mathrm{~Hz}$ repetition rate; $(\mathbf{B})$ evaluation of $\mathrm{Ag}-\mathrm{NP}$ stability produced at $30 \mathrm{~Hz}$ repetition rate in aqueous starch solution over a period of I month (samples stored at ambient temperature in the dark). Generally, in aqueous starch solution, good stability is achieved and is retained contrary to colloids produced in water.

Abbreviation: $\mathrm{Ag}-\mathrm{NP}$, silver nanoparticles. 
in the presence of starch. Finally, Figure 9B confirms that during the initial days post-LASiS, a further accumulation of negatively charged ions and molecules in the nanoparticle corona occurs as indicated by an increase in negative zeta potential. Illustrative for post-LASiS aftereffects is the report by Mafuné and coworkers that Ag-NPs can continue to attract emitted material and grow in pure water for several days after LASiS, ${ }^{31}$ mainly because small particulate materials, such as ions, have long lifetimes in solution and show a high propensity to coalesce. In our case, a stabilizing agent covers the Ag-NP surface, which should effectively prevent ions and particles from the surrounding environment from reaching the stabilized nanoparticle. However, rearrangements cannot be discounted because of the unique constellation in starch, with a helical and highly branched component, and there seems to be some indication that this is true for cluster formation. That generally no further particle growth occurs over 30 days of storage is shown by the lack of red shift in the $400 \mathrm{~nm}$ peak (Figure 8), so continued particle growth may effectively be excluded.

Over time, some negative charge is lost, but the zeta potential never drops below $-50 \mathrm{mV}$ and the solution remains stabile, as illustrated for the $30 \mathrm{~Hz}$ repetition rate in Figure $8 \mathrm{~B}$. This is so for all repetition rates, except for $10 \mathrm{~Hz}$, which produces incipiently unstable colloids based on zeta potential measurements. Silver nanoparticles produced in pure water generally only reach moderate stability and deteriorate over time to form significantly unstable colloidal solutions (Figure 9B).

In general, the results of our stability measurements show that a good measure of colloidal stability is present and retained, but that some rearrangements occur. These are probably both dependent on the initial mean size and size distribution as determined by the repetition rate and the unique properties of starch with its two very distinct forms and high polymerization degree.

\section{Conclusion}

Generally, nanoparticle synthesis via laser ablation in solution offers a number of advantages over more conventional physical and chemical methods, including a smaller average size, smaller size distributions, and the fact that LASiS does not require the use of hazardous chemicals. Furthermore, LASiS-based techniques are easy in their experimental setup and execution and may be suitable for concomitant synthesis and in situ biofunctionalization. ${ }^{37}$ Recent years have seen a small boom in LASiS-based publications and numerous research groups have devoted their time to developing
LASiS as a technique. However, despite all these efforts, a comprehensive understanding of the fundamental processes underlying LASiS is still lacking to such an extent that there is a large unrealized potential for the controlled production of nanoparticles with this technique. Therefore, it is unquestionably necessary to investigate the influence of both the synthesis medium and the laser settings on the size, size distribution, shape, purity, and functionalization of the final nanoparticle product. This is highly important, since the quality and applicability of nanoparticles is directly determined by their stability, hydrodynamic size, purity, and surface chemistry, which are especially important in biomedical and biological applications.

In order to stabilize and afford some control over size and shape, several groups, including ours, started to use nontoxic natural stabilizing agents in aqueous solution in combination with LASiS for the production of metallic nanoparticles. In this paper we confirm our previous results ${ }^{18}$ and prepared starch-stabilized Ag-NPs via LASiS. Here we investigated the effect of laser repetition rate on the particle size and size distribution, and particle stability over time in an experimental setup that limits fragmentation and most likely promotes more continuous growth. Our results show that with increasing repetition rates to a maximum of $40 \mathrm{~Hz}$, both the mean size and the size distribution increases significantly. The mean particle size scales near linearly with the repetition rate in both starch solution and neat water, albeit that in water this scaling evolves faster. That the particle size increases with the repetition rate is most likely a direct result of the experimental setup, which reduces the probability that fragmentation occurs, since this phenomenon is also observed in neat water and not just the starch solution. The mean particle size in starch solution always remained below $16 \mathrm{~nm}$, in stark contrast to particles prepared in neat water, which were larger with an average size of $\sim 30 \mathrm{~nm}$ and showed broad size distributions. The smallest mean particle size of $\sim 10 \mathrm{~nm}$ was achieved at $10 \mathrm{~Hz}$ repetition rate with a narrow bandwidth of $6 \mathrm{~nm}$ FWHM. Furthermore, in starch LASiS, large clusters were strikingly absent, in stark contrast to LASiS in water, in which agglomeration of different sized particles was clearly noticed, as might be expected. The fact that in starch LASiS larger globular particles were observed indicates that more controlled particle growth occurs from the initial nucleation in the presence of the stabilizer. Most importantly, starch prevented individual particles from agglomerating to larger clusters during LASiS, which confirms our previous considerations and results. ${ }^{18}$ Furthermore, if fragmentation occurred, this would be most efficient at low 
repetition rates, because a higher fraction of not-previously irradiated nanoparticles in the colloidal solution is irradiated. This might to some extent explain the small average size, narrow size distribution, and absence of clusters at the $10 \mathrm{~Hz}$ repetition rate. Furthermore, we hypothesize that the particle growth occurs within the helical structure of amylose and that the necessary matter for growth is recruited from the environment. The diameter of the helix can be dynamically adaptable and thus such considerations are entirely within the realm of what is possible. Since experiments with starch-coated SWCNT have shown that these become insoluble in the absence of amylopectin, we suspect that this amylose-Ag-NP complex is coated with amylopectin in an extensive network that affords steric hindrance and prevents agglomeration.

Stability measurements show that the Ag-NP-starch composite remains stabile after prolonged storage for up to 1 month. The mean particle size does not significantly change and UV-vis spectroscopy showed only minor deviations in the spectrum, which indicates that no significant aging occurs. Zeta potential measurements show that a good measure of colloidal stability is present and retained over time. In contrast, particles produced in water only reach moderate stability and deteriorate over time to form significantly unstable colloidal solutions, which was confirmed through TEM evaluation of stored solutions. The optimum initial and long-term stability of the Ag-NP-starch composite is achieved at the $30 \mathrm{~Hz}$ repetition rate. Consequently, Ag-NP synthesis via LASiS in starch solutions is a trade-off between small size and narrow size distributions and inherent and long-term stability.

Since many questions remain, we initiated continuative research to investigate the influence of repetition rate and various other laser parameters on the LASiS-based synthesis of nanoparticles in the presence of starch. It will be essential to determine the effect of the individual starch components (amylose and amylopectin) on LASiS of various types of metal nanoparticles. Finally, our experimental results not only show that the synthesis environment, the type of stabilizer used, and its chemical and structural properties, but also that variation of one single laser parameter drastically determines the synthesis outcome and final product.

\section{Acknowledgments}

This work was supported by the Universiti Putra Malaysia postdoctoral research fellowship scheme (Dr Zamiri) and a Grant for External Collaborative Research NSR-8978 (Dr Drummen).

\section{Disclosure}

Dr Drummen is partially exempted from his duties by Bio\&Nano-Solutions to pursue fundamental scientific research. The authors declare no further conflicts of interest.

\section{References}

1. Henglein A. Small-particle research - Physicochemical properties of extremely small colloidal metal and semiconductor particles. Chem Rev. 1989;89(8):1861-1873.

2. Henglein A. Physicochemical properties of small metal particles in solution - Microelectrode reactions, chemisorption, composite metal particles, and the atom-to-metal transition. J Phys Chem. 1993;97(21):5457-5471.

3. Niemeyer CM. Nanoparticles, proteins, and nucleic acids: Biotechnology meets materials science. Angew Chem Int Ed Engl. 2001;40(22): 4128-4158.

4. Roduner E. Size matters: why nanomaterials are different. Chem Soc Rev. 2006;35(7):583-592.

5. Schmid G. Large clusters and colloids - Metals in the embryonic state. Chem Rev. 1992;92(8):1709-1727.

6. Eustis S, Krylova G, Eremenko A, Smirnova N, Schill AW, El-Sayed M. Growth and fragmentation of silver nanoparticles in their synthesis with a fs laser and $\mathrm{CW}$ light by photo-sensitization with benzophenone. Photochem Photobiol Sci. 2005;4(1):154-159.

7. Anderson HH, editor. Small Particles and Inorganic Clusters. Berlin, Heidelberg, Germany: Springer Verlag; 1997. ISSPIC 8.

8. Jain PK, Lee KS, El-Sayed IH, El-Sayed MA. Calculated absorption and scattering properties of gold nanoparticles of different size, shape, and composition: Applications in biological imaging and biomedicine. J Phys Chem B. 2006;110(14):7238-7248.

9. Munro CH, Smith WE, Garner M, Clarkson J, White PC. Characterization of the surface of a citrate-reduced colloid optimized for use as a substrate for surface-enhanced resonance Raman scattering. Langmuir. 1995;11(10):3712-3720.

10. Liu J, Li X, Zeng X. Silver nanoparticles prepared by chemical reduction-protection method, and their application in electrically conductive silver nanopaste. J Alloys Compd. 2010;494(1-2):84-87.

11. Milani P, Deheer WA. Improves pulsed laser vaporization source for production of intense beams of neutral and ionized clusters. Rev Sci Instrum. 1990;61(7):1835-1838.

12. Amendola V, Meneghetti M. Laser ablation synthesis in solution and size manipulation of noble metal nanoparticles. Phys Chem Chem Phys. 2009;11(20):3805-3821.

13. Fojtik A, Henglein A. Laser ablation of films and suspended particles in a solvent - formation of cluster and colloid solutions. Berichte der Bunsen-Gesellschaft. 1993;97(2):252-254.

14. Neddersen J, Chumanov G, Cotton TM. Laser-ablation of metals a new method for preparing SERS active colloids. Appl Spectrosc. 1993;47(12):1959-1964.

15. Sibbald MS, Chumanov G, Cotton TM. Reduction of cytochrome c by halide-modified, laser-ablated silver colloids. J Phys Chem. 1996; 100(11):4672-4678.

16. Rao CRK, Lakshminarayanan V, Trivedi DC. Synthesis and characterization of lower size, laurylamine protected palladium nanoparticles. Mater Lett. 2006;60(25-26):3165-3169.

17. Huang NM, Radiman S, Lim HN, et al. gamma-Ray assisted synthesis of silver nanoparticles in chitosan solution and the antibacterial properties. Chem Eng J. 2009;155(1-2):499-507.

18. Zamiri R, Azmi BZ, Darroudi M, et al. Preparation of starch stabilized silver nanoparticles with spatial self-phase modulation properties by laser ablation technique. Appl Phys A. 2011;102:189-194.

19. He F, Zhao DY. Preparation and characterization of a new class of starch-stabilized bimetallic nanoparticles for degradation of chlorinated hydrocarbons in water. Environ Sci Technol. 2005;39(9):3314-3320. 
20. Janssen L, Moscicki L, editors. Thermoplastic Starch: A Green Material for Various Industries. 1st ed. Weinheim, Germany: Wiley-VCH Verlag $\mathrm{GmbH}$ and $\mathrm{Co}, \mathrm{KGaA} ; 2009$.

21. Deka J, Paul A, Ramesh A, Chattopadhyay A. Probing Au nanoparticle uptake by enzyme following the digestion of a starch-Au-nanoparticle composite. Langmuir. 2008;24(18):9945-9951.

22. Cui H, Liu P, Yang GW. Noble metal nanoparticle patterning deposition using pulsed-laser deposition in liquid for surface-enhanced Raman scattering. Appl Phys Lett. 2006;89(15):153124-153123.

23. Pecharroman C. Influence of the close sphere interaction on the surface plasmon resonance absorption peak. Phys Chem Chem Phys. 2009;11(28):5922-5929.

24. Kerker M. The optics of colloidal silver - Something old and something new. J Colloid Interface Sci. 1985;105(2):297-314.

25. Brause R, Moltgen H, Kleinermanns K. Characterization of laser-ablated and chemically reduced silver colloids in aqueous solution by UV/VIS spectroscopy and STM/SEM microscopy. Appl Phys B-Lasers Opt. 2002;75(6-7):711-716.

26. Mafuné F, Kohno J, Takeda Y, Kondow T, Sawabe H. Formation of gold nanoparticles by laser ablation in aqueous solution of surfactant. J Phys Chem B. 2001;105(22):5114-5120.

27. Menéndez-Manjón A, Barcikowski S. Hydrodynamic size distribution of gold nanoparticles controlled by repetition rate during pulsed laser ablation in water. Appl Surf Sci. 2011;257(9): 4285-4290.

28. Sylvestre J-P, Kabashin AV, Sacher E, Meunier M, Luong JHT. Stabilization and Size Control of Gold Nanoparticles during Laser Ablation in Aqueous Cyclodextrins. J Am Chem Soc. 2004;126(23): 7176-7177.
29. Tsuji T, Thang DH, Okazaki Y, Nakanishi M, Tsuboi Y, Tsuji M. Preparation of silver nanoparticles by laser ablation in polyvinylpyrrolidone solutions. Appl Surf Sci. 2008;254(16):5224-5230.

30. Zamiri R, Azmi BZ, Sadrolhosseini AR, Ahangar HA, Zaidan AW, Mahdi MA. Preparation of silver nanoparticles in virgin coconut oil using laser ablation. Int J Nanomedicine. 2011;6:71-75.

31. Mafuné F, Kohno J, Takeda Y, Kondow T, Sawabe H. Formation and size control of silver nanoparticles by laser ablation in aqueous solution. $J$ Phys Chem B. 2000;104(39):9111-9117.

32. Yang GW. Laser ablation in liquids: Applications in the synthesis of nanocrystals. Prog Mater Sci. 2007;52(4):648-698.

33. Pérez S, Bertoft E. The molecular structures of starch components and their contribution to the architecture of starch granules: A comprehensive review. Starch-Starke. 2010;62(8):389-420.

34. Lehmann J. Carbohydrates: Structure and Biology. Stuttgart, Germany: Thieme Medical Publishers; 1998.

35. Takeo K, Tokumura A, Kuge T. Complexes of starch and its related materials with organic compounds. 10. X-ray-diffraction of amylose fatty acid complexes. Starch-Starke. 1973;25(11):357-362.

36. Star A, Steuerman DW, Heath JR, Stoddart JF. Starched carbon nanotubes. Angew Chem Int Ed Engl. 2002;41(14):2508-2512.

37. Petersen S, Barcikowski S. In situ boconjugation: Single step approach to tailored nanoparticle-bioconjugates by ultrashort pulsed laser ablation. Adv Funct Mater. 2009;19(8):1167-1172.

38. Hunter RJ. Foundations of Colloid Science. 2nd ed. Oxford, UK: Oxford University Press; 2001

39. Hunter RJ. Zeta potential in colloid science: Principles and applications. London, UK: Academic Press Inc; 1988.
International Journal of Nanomedicine

\section{Publish your work in this journal}

The International Journal of Nanomedicine is an international, peerreviewed journal focusing on the application of nanotechnology in diagnostics, therapeutics, and drug delivery systems throughout the biomedical field. This journal is indexed on PubMed Central, MedLine, CAS, SciSearch $\AA$, Current Contents ${ }^{\circledR} /$ Clinical Medicine,

\section{Dovepress}

Journal Citation Reports/Science Edition, EMBase, Scopus and the Elsevier Bibliographic databases. The manuscript management system is completely online and includes a very quick and fair peer-review system, which is all easy to use. Visit http://www.dovepress.com/ testimonials.php to read real quotes from published authors. 